\title{
Investimento Materno e História Reprodutiva de Mães Residentes em Contextos com Diferentes Graus de Urbanização ${ }^{1}$
}

\author{
Viviane Vieira \\ Lílian Costa Silveira \\ Mauro Luís Vieira² \\ Universidade Federal de Santa Catarina \\ Alessandra Bonassoli Prado \\ Universidade de São Paulo
}

\begin{abstract}
RESUMO - A presente pesquisa tem como objetivo caracterizar o investimento materno e a história reprodutiva de mães que vivem em diferentes contextos. Para tal, 150 mães que residiam em três contextos com diferentes graus de urbanização foram entrevistadas. Por meio da análise estatística dos dados constatou-se que houve diferenças significativas entre os contextos, além de correlações positivas entre os núcleos reprodutivos, como idade da primeira relação sexual e idade da mãe no nascimento do primeiro filho. Conclui-se que as estratégias reprodutivas são influenciadas pela história de vida das mães, das condições sociodemográficas atuais e do contexto onde vivem.
\end{abstract}

Palavras-chave: teoria da história de vida; estratégias reprodutivas; urbanização; investimento materno.

\section{Maternal Investment and Reproductive History of Mothers Living in Contexts with Different Degrees of Urbanization}

\begin{abstract}
The present study has the purpose to characterize both the maternal investment and the reproductive history of mothers from different contexts. To this end, 150 mothers living in three contexts with different degrees of urbanization were interviewed. Through statistical analysis of the data, it was found that there were significant differences between contexts as well as positive correlations between reproductive cores, such as age at first sexual intercourse and mother' age at the birth of the first child. It was concluded that reproductive strategies are influenced by the mothers' life history, current sociodemographic conditions, and the context where they live.
\end{abstract}

Keywords: life history theory; reproductive strategies; urbanization; maternal investment.

Uma teoria que integre a biologia e a cultura é a que mais se aproxima de explicar satisfatoriamente os fenômenos da universalidade e da diversidade no desenvolvimento humano. O modo como a base biológica e a cultura interagem para formar indivíduos diferenciados deve ser estudado no ciclo de vida completo (Keller, 1996, 2002). O ciclo de vida é relacionado à sobrevivência das espécies, que envolve também o comportamento reprodutivo, e está relacionado não apenas com a geração de novos descendentes, mas também com o cuidado da prole, principalmente em mamíferos (Bjorklund \& Pellegrini, 2000; Geary, 2000; Geary \& Flynn, 2001).

$\mathrm{Na}$ compreensão da perspectiva evolucionista, a função central do cuidado parental e da família humana é promover um contexto favorável para a melhor elaboração de competências sociais complexas (empatia, desconfiança, colaboração, competição) e, por conseguinte, o desenvolvimento infantil e a transformação da criança em um adulto hábil para enfrentar as adversidades da fase adulta (Bjorklund \& Pellegrini, 2000; Davis \& Daly, 1997; Geary, 2000; Geary \& Flynn, 2001). O contexto de desenvolvimento humano

1 Apoio: $\mathrm{CNPq}$

2 Endereço para correspondência: UFSC - $\mathrm{CFH}$, Campus Universitário, Departamento de Psicologia, Trindade. Florianópolis, SC. CEP 88049900. Fone: (48) 3721-9984.E-mail: maurolvieira@gmail.com. envolve diversos aspectos que contribuem para o incremento de diferentes competências individuais dos descendentes. Um dos aspectos que se destaca é o repertório comportamental dos pais para a resolução de diferentes problemas e objetivos, principalmente os referentes ao processo de amadurecimento (Geary, 2000; Geary \& Flynn, 2001).

O esforço parental relaciona-se com o desenvolvimento físico e psicológico da prole. Na Teoria do Investimento Parental, os esforços reprodutivos dos indivíduos estão relacionados com seus esforços somáticos. Embora a espécie humana seja uma espécie de alto investimento parental, diferenças quantitativas podem ser compreendidas de duas maneiras: o primeiro caracteriza o indivíduo que na infância experimenta um ambiente com escassez de recursos, baixo investimento parental e clima familiar instável ou conturbado; já o segundo descreve o indivíduo que, vivendo uma situação de abundância de recursos, é beneficiário de um alto investimento parental e um clima familiar estável, seguro e positivo (Hill \& Kaplan, 1999; Hoier, 2002; Maestripieri, Roney, DeBias, Durante \& Spaepen, 2004; MacDonald, 1997; Mace, 2000).

Ao lado de consequências psicológicas nas relações de apego entre criança e pais, esses dois tipos de experiências levariam a diferenças no início da puberdade. Sugere-se que, 
no primeiro caso (baixo investimento), o amadurecimento acontecerá mais cedo do que no segundo caso (alto investimento) e seguirá uma trajetória reprodutiva diferenciada; seu estilo reprodutivo pode ser caracterizado como quantitativo (mais filhos, menor espaçamento entre os nascimentos, mais parceiros, menor idade na primeira relação sexual e baixo investimento parental). O segundo modelo, ao contrário, seria mais qualitativo (menos e mais espaçados filhos e alto investimento parental, menos parceiros sexuais e maior idade na idade da primeira relação sexual) (Keller, 1996; MacDonald, 1997; Mace \& Sear, 1997). Esse direcionamento do investimento parental envolve os custos e benefícios que cada uma das estratégias reprodutivas contém, especialmente no que se refere à energia despendida (Hill \& Kaplan, 1999; Hoier, 2002; MacDonald, 1997; Mace, 2000; Maestripieri, Roney, DeBias, Durante \& Spaepen, 2004).

Uma teoria que se propõe a explicar os eventos da vida relacionados com a estratégia de reprodução (número de parceiros sexuais, primeiro filho, quantidade de filhos), crescimento e mortalidade é a da História de Vida (Hill \& Kaplan, 1999; Hoier, 2002; MacDonald, 1997). Esta teoria parte da suposição que o tempo, o esforço e a energia utilizados em uma atividade diminuem a probabilidade de investir em outra.

A Teoria da História de Vida enfatiza negociações (trade$o f f s$ ) envolvendo custos e benefícios de esforços somáticos (crescimento e manutenção corporal) e reprodutivos. Os recursos de tempo e energia de um organismo podem ser investidos: (a) para garantir a integridade física por meio do esforço somático, que compreende todo investimento de um organismo para seu crescimento, desenvolvimento e manutenção e, desse modo, acumular potencial reprodutivo; (b) na procura e disputa por parceiros reprodutivos aptos a procriar em um esforço de acasalamento; e (c) no esforço parental necessário para a progênie atingir a maturidade e se reproduzir, o que se relaciona também com o tamanho da prole. Uma progênie grande aumenta o sucesso reprodutivo, desde que não esteja acima das condições físicas do progenitor ou dos recursos oferecidos pelo ambiente. Por outro lado, uma prole pequena e saudável é mais adequada quando as condições do progenitor e os recursos do ambiente são limitados (Geary \& Flynn, 2001; Hill \& Kaplan, 1999; Hoier, 2002; Hrdy, 1999/2001; Simpson \& Gangestad, 2001; Vigil, Geary \& Byrd-Craven, 2005; Voland, 1998).

As unidades fundamentais de análise da Teoria da História de Vida são a maturação e a reprodução. Nesse sentido, algumas características que definem o ciclo de vida analisado são: tamanho corporal, maturidade sexual e idade da primeira reprodução, intervalo entre nascimentos, tamanho da prole e idade no desmame. A partir desses dados, pode-se notar que as estratégias reprodutivas envolvem a idade da menarca, primeira gestação, quantidade de parceiros sexuais e de filhos, características que podem interferir na dinâmica do cuidado parental e na alocação de tempo e recursos (Ellis \& Garber, 2000; Hill \& Kaplan, 1999; MacDonald, 1997).

$\mathrm{O}$ ambiente onde o indivíduo está inserido fornece sinais e recursos que auxiliam em sua interpretação sobre quais estratégias são mais vantajosas, considerando que a experiência prévia - incluindo o cuidado parental recebido -, os padrões culturais locais, eventos estressores durante a vida e acontecimentos inesperados podem intervir nessa leitura (Hill
\& Kaplan, 1999; MacDonald, 1997; Maestripieri \& cols., 2004; Quinlan, 2003). Mesmo quando se refere à estratégia reprodutiva, as experiências negativas e estressantes durante a infância podem reduzir a oportunidade de acurar o potencial reprodutivo, restringindo opções de reprodução individual e competências quando adultos (Mace, 2000; Maestripieri \& cols., 2004; Quinlan, 2003).

A partir das ideias expostas até aqui, é objetivo desta pesquisa relacionar as condições de vida na infância das mães participantes com variáveis referentes a investimento parental e à adoção de estratégias reprodutivas. Utilizando como referência a Teoria da História de Vida, a hipótese deste estudo é a de que a adoção de uma estratégia mais quantitativa (menarca mais cedo, primeira relação mais cedo, mais parceiros e mais filhos) se relaciona positivamente com condições de vida familiar estressantes na infância da mãe. Por outro lado, o uso de uma estratégia mais qualitativa (menarca mais tarde, primeira relação mais tarde, menos parceiros e menos filhos) se relaciona positivamente com condições de vida familiar com baixo nível de estresse durante a infância das mães participantes.

Além disso, por meio da presente pesquisa procura-se investigar as semelhanças e diferenças nas estratégias reprodutivas em três contextos catarinenses: (a) Capital - cidade com quase 400 mil habitantes e alto nível de urbanização; (b) Interior-Leste - duas cidades no norte do estado, uma próxima da outra, com menos de 20 mil habitantes e nível de urbanização em desenvolvimento; e (c) Interior-Oeste cidade de menos de 20 mil habitantes, mas com um baixo nível de urbanização.

\section{Método}

\section{Participantes $^{3}$}

Participaram do estudo 150 mães, sendo 50 residentes em cada um dos contextos constituintes da amostra. As participantes deveriam ter a partir de 20 anos de idade, com pelo menos um filho com idade entre 0 e 6 anos de idade. A amostra do contexto chamado Capital foi composta por mães com idade entre 21 e 49 anos $(M=32,14 ; D P=6,37)$, número de filhos entre um e quatro, sendo que a maioria tinha um filho $(\mathrm{n}=30)$ no período da coleta de dados. A maior parte dessas mães era casada ou vivia em união estável ( $\mathrm{n}=$ 42), com composição familiar nuclear $(\mathrm{n}=37)$, era católica $(n=26)$, tinha trabalho remunerado $(n=39)$, foi criada na zona urbana $(n=42)$, tinha escolaridade superior $(n=36)$ e tinha renda mensal familiar acima de $\mathrm{R} \$ 2.000,00(\mathrm{n}=35)$.

No contexto Interior-Leste, a amostra foi composta por mães residentes nas cidades de Corupá e Schroeder, com idade entre 21 e 38 anos $(M=29,16 ; \mathrm{DP}=3,82)$, total de filhos entre um e quatro, sendo que a maioria tinha um $(n=28)$ ou dois filhos $(\mathrm{n}=17)$ no período da coleta de dados. A maior

3 Esse estudo faz parte de uma pesquisa nacional, do Instituto do Milênio, denominada Investimento e cuidado parentais: aspectos biológicos, ecológicos e culturais e valores parentais, realizada em seis estados brasileiros. Nesse projeto, a amostra geral foi composta por 606 mães. 
parte dessas mães era casada ou vivia em união estável ( $\mathrm{n}=$ 47), com composição familiar nuclear $(\mathrm{n}=44)$, era católica $(\mathrm{n}=33)$, foi criada na zona rural $(\mathrm{n}=28)$, tinha trabalho remunerado $(n=42)$, escolaridade entre médio completo e superior incompleto $(\mathrm{n}=27)$ e tinha renda mensal familiar entre $R \$ 301,00$ e $R \$ 1.300,00(n=35)$.

No contexto Interior-Oeste, a amostra era composta por mães residentes na cidade de Guaraciaba, com idade entre 20 e 43 anos $(M=29,80 ; D P=6,06)$, total de filhos entre um e cinco, sendo que a maioria tinha um $(\mathrm{n}=15)$ ou dois filhos $(\mathrm{n}=21)$ no período da coleta de dados. A maior parte dessas mães era casada ou vivia em união estável $(\mathrm{n}=47)$, com composição familiar nuclear $(\mathrm{n}=41)$, era católica $(\mathrm{n}=44)$, não trabalhava $(\mathrm{n}=33)$, foi criada na zona rural $(\mathrm{n}=34)$, tinha escolaridade até ensino fundamental completo $(\mathrm{n}=32)$ e renda mensal familiar de até $\mathrm{R} \$ 1.000,00(\mathrm{n}=34)$.

\section{Ambiente}

Os três contextos nos quais foram realizadas as coletas de dados receberam a classificação de Capital, Interior-Leste e Interior-Oeste. Todos situam-se no Estado de Santa Catarina, região Sul do Brasil.

O contexto chamado Capital situa-se no litoral de Santa Catarina, a capital do estado. De colonização açoriana, Florianópolis tem como principal atividade econômica o turismo e o comércio, destacando-se também como polo tecnológico no estado. Com 396.723 habitantes, em termos gerais de infraestrutura, a cidade conta com 354 estabelecimentos de saúde, sendo 73 públicos; 3.784 creches/centros de educação infantil frequentadas por 176.316 crianças de 0 a 6 anos de idade; universidades; bibliotecas e áreas culturais e de lazer (IBGE, 2006; Governo do Estado de Santa Catarina, 2007). Esse contexto tem alto nível de urbanização.

O contexto do Interior-Leste contou com a participação de duas cidades no interior do estado, agrupadas para fins de coleta de dados em um único contexto por serem semelhantes em vários aspectos. O município de Corupá é uma cidade de colonização alemã, italiana e polonesa, com população estimada de 12.758 habitantes em 2007 (IBGE, 2006). Segundo dados do Governo do Estado de Santa Catarina (2007), o comércio e a indústria são responsáveis por mais da metade da renda do município, que é o maior produtor de banana do Estado. Em termos de infraestrutura, a cidade possui oito estabelecimentos de saúde, sem leitos hospitalares. Em 2006 havia 401 crianças na Pré-Escola e 1.779 no Ensino Fundamental (IBGE, 2006).

O município de Schroeder, com 12.776 habitantes (IBGE, 2006), também é de colonização alemã. A economia do município gira em torno da agropecuária (Governo do Estado de Santa Catarina, 2007). Em termos de infraestrutura, a cidade dispõe de seis estabelecimentos de saúde, sem leitos hospitalares. Em 2006 havia 235 crianças matriculadas na Pré-Escola e 1.342 no Ensino Fundamental (IBGE, 2006). Nesse contexto Interior-Leste, o nível de urbanização está em desenvolvimento, com uma proximidade territorial com os polos da indústria metal-mecânica de Santa Catarina (Joinville e Jaraguá do Sul).
Finalmente, o contexto denominado Interior-Oeste situa-se no extremo oeste de Santa Catarina, na cidade de Guaraciaba. De colonização alemã e italiana, Guaraciaba tem 10.604 habitantes, oito estabelecimentos de saúde, 289 crianças na Pré-Escola e 1.554 no Ensino Fundamental (IBGE, 2006), e sua atividade principal é a agropecuária (Governo do Estado de Santa Catarina, 2007). Nesse contexto, o nível de urbanização é baixo, predominando as áreas destinadas a lavouras e pastagens.

\section{Instrumento}

O instrumento foi constituído, primeiramente, por uma parte de caracterização sociodemográfica, seguido por um questionário de história reprodutiva e dados sobre sexualidade. Incluía perguntas sobre a menarca, idade da primeira relação sexual, o número de parceiros sexuais até o momento atual, número de filhos, idade no nascimento do primeiro filho, número de casamentos, idade no primeiro relacionamento amoroso e duração desse relacionamento.

O instrumento também continha uma parte sobre a qualidade de vida familiar passada (infância). Essa parte continha perguntas sobre o clima familiar (de "muito tranquilo" a "muito tumultuado"), relação do casal (de "nada conflituosa" a "extremamente conflituosa") e afetividade (de "nada afetuosa" a "extremamente afetuosa"). As respostas iam de 1 a 5 pontos, em que o número 3 era o ponto médio. Com isso, media-se o Índice da Qualidade do Ambiente Familiar Passado; quanto mais próximo de 5 (com exceção da afetividade que era invertido), menor a qualidade do ambiente familiar e, consequentemente, o ambiente era considerado mais estressante.

Por fim, havia uma questão sobre as condições materiais durante a infância, ou seja, a organização financeira e material da família quando a mãe era criança. A resposta poderia ir de "Vivia muito bem" (1) a "Passei muita necessidade" (5), em que o número 3 indicava um ponto médio.

\section{Procedimento}

O contato inicial com as mães foi feito por meio de creches, centros de educação infantil e postos de saúde. Além disso, as mães participantes indicavam outras para a pesquisa. As entrevistas, com duração média de 1 hora, eram agendadas em contato prévio com as mães, que escolhiam o local, a data e o horário de sua preferência.

Sobre os cuidados éticos, a pesquisa foi aprovada pelo Conselho de Ética da Universidade Federal de Santa Catarina, protocolada sob o número 239/06. As mães participantes assinaram um Termo de Consentimento Livre e Esclarecido, com todas as informações necessárias acerca da pesquisa, inclusive dados para contato com os pesquisadores.

\section{Análise de dados}

Os dados obtidos nos três contextos foram analisados por meio do programa estatístico para Windows, primeiramente 
em conjunto, em que foram comparadas as médias a partir da análise de variância (ANOVA), que mostrava se havia diferenças entre elas, e do teste post hoc de Tukey, para localizar essas diferenças (Dancey \& Reidy, 2006).

Os três contextos desse estudo também foram analisados separadamente, por meio de análise de correlações, em que o teste $r$ de Pearson mostra o grau de relacionamento entre as variáveis e a probabilidade dessas relações ocorrerem por erro amostral (Dancey \& Reidy, 2006).

\section{Resultados}

$\mathrm{Na}$ análise conjunta dos três contextos foi possível verificar que, em relação à idade da menarca e à idade da primeira relação sexual, não houve diferença estatisticamente significativa entre os contextos. No entanto, quando comparados os intervalos entre a idade da respondente na primeira relação sexual e a idade da mesma no nascimento do primeiro filho, as diferenças entre esses intervalos foram estatisticamente significativas entre Capital, Interior-Leste e Interior-Oeste.

Quanto ao número de parceiros sexuais, a diferença entre as médias dos contextos foi significativa somente quando comparados com a Capital (Capital/Interior- Oeste: $\mathrm{p}<0,001$; Capital e Interior-Leste: $\mathrm{p}<0,001)$. No que se refere ao número de filhos, a diferença entre as médias dos contextos foi significativa somente quando comparados com o InteriorOeste (Interior-Oeste e Capital: $\mathrm{p}<0,001$; Interior-Oeste e Interior-Leste: $\mathrm{p}<0,005)$. Em relação à idade da respondente no nascimento do primeiro filho, a diferença entre as médias dos contextos foi significativa somente quando comparados com a Capital (Capital e Interior-Oeste: $\mathrm{p}<0,001$; Capital e Interior-Leste: $\mathrm{p}<0,05)$.

$\mathrm{Na}$ análise dos contextos realizada separadamente, as variáveis consideradas foram selecionadas a partir do estudo da Teoria da História de Vida, que incluem dados sociodemográficos, bem como dados da trajetória reprodutiva. Em todos os contextos, condições materiais menos favoráveis na infância apareceram associadas com um ambiente com menor qualidade familiar passada nesse mesmo período. Além disso, uma correlação positiva entre a idade da primeira relação sexual e a idade em que a mãe teve o primeiro filho também apareceu nos três contextos.

Além de associações comuns entre Capital, InteriorLeste e Interior-Oeste, houve também algumas correlações em dois desses três contextos. No Interior-Oeste e Capital, a variável qualidade do ambiente familiar passado apareceu positivamente relacionada com dois núcleos da trajetória reprodutiva (idade da primeira relação sexual e número de parceiros). Em relação aos dados sociodemográficos, um nível maior de renda familiar mensal esteve associado com menor número de parceiros sexuais e idade mais tardia da mãe no nascimento do primeiro filho. Já níveis mais altos de escolaridade apareceram correlacionados com idade mais avançada da mãe no nascimento do primeiro filho, nos contextos Interior-Leste e Capital.

As tabelas 1, 2 e 3 mostram as correlações entre dados sociodemográficos e indicadores do núcleo da história reprodutiva de mães nos contextos investigados. Conforme indica a Tabela 1, no contexto Capital, as variáveis escolaridade e renda familiar mensal se correlacionaram de modo significativo e positivamente com dados da história reprodutiva que indicam uma estratégia reprodutiva mais qualitativa, como menor número de parceiros sexuais, menor número de filhos, maior idade na primeira relação sexual e no nascimento do primeiro filho.

Também é possível verificar, na Tabela 1, que um índice baixo de qualidade do ambiente familiar na infância se relacionou com a adoção de uma estratégia mais quantitativa no que se refere à reprodução, como mostram as correlações entre as variáveis qualidade do ambiente familiar passado, número de parceiros sexuais e idade da primeira relação sexual.

Assim como no contexto Capital, uma escolaridade mais alta esteve associada com maior idade da mãe no nascimento do primeiro filho, no contexto Interior-Leste, como demonstrado na Tabela 2. No entanto, a renda não teve associações estatisticamente significativas com nenhum dos dados da história reprodutiva. Entre os núcleos de trajetória reprodutiva, houve uma correlação positiva entre a idade da mãe na primeira relação sexual e no nascimento do primeiro filho.

Assim como nos anteriores, o contexto Interior-Oeste, como pode ser observado na Tabela 3 , também apresentou associação de escolaridade e renda com núcleos reprodutivos (número de filhos, idade da mãe no nascimento do primeiro filho, número de parceiros e quantidade de filhos total da participante), sendo que a renda foi a variável sociodemográfica que mais esteve associada aos dados da trajetória reprodutiva.

Também no Interior-Oeste apareceram correlações entre quatro dos cinco núcleos reprodutivos. A idade na primeira relação sexual e o número de filhos apresentaram uma correlação estatisticamente significativa com a idade mais tardia da mãe no nascimento do primeiro filho. $\mathrm{O}$ número de parceiros também teve uma correlação positiva com um maior número de filhos. Ainda em relação a esses quatro núcleos da história reprodutiva, a variável referente às condições materiais na infância apareceu associada a todos eles, indicando que condições materiais desfavoráveis na infância da mãe se relacionam com estratégias de ação reprodutiva mais quantitativa.

Sobre a menarca, essa foi a única variável que não teve associação estatisticamente significativa com os dados sociodemográficos. Em relação aos demais núcleos da história reprodutiva, apenas no contexto Capital constatou-se que houve correlação entre a menarca e o número de parceiros, mostrando que quanto mais tarde ocorreu a menarca, maior o número de parceiros sexuais da respondente até o momento da entrevista, não correspondendo ao que foi encontrado na literatura.

\section{Discussão}

A partir dos resultados obtidos, a hipótese que propõe uma relação das estratégias reprodutivas adotadas com ambientes estressantes e com baixa disponibilidade de recursos foi parcialmente confirmada. A ausência de associações com a menarca vai de encontro com a hipótese inicial. Contudo, esse núcleo já apresentava resultados contraditórios na literatura; enquanto alguns pesquisadores encontram relações 


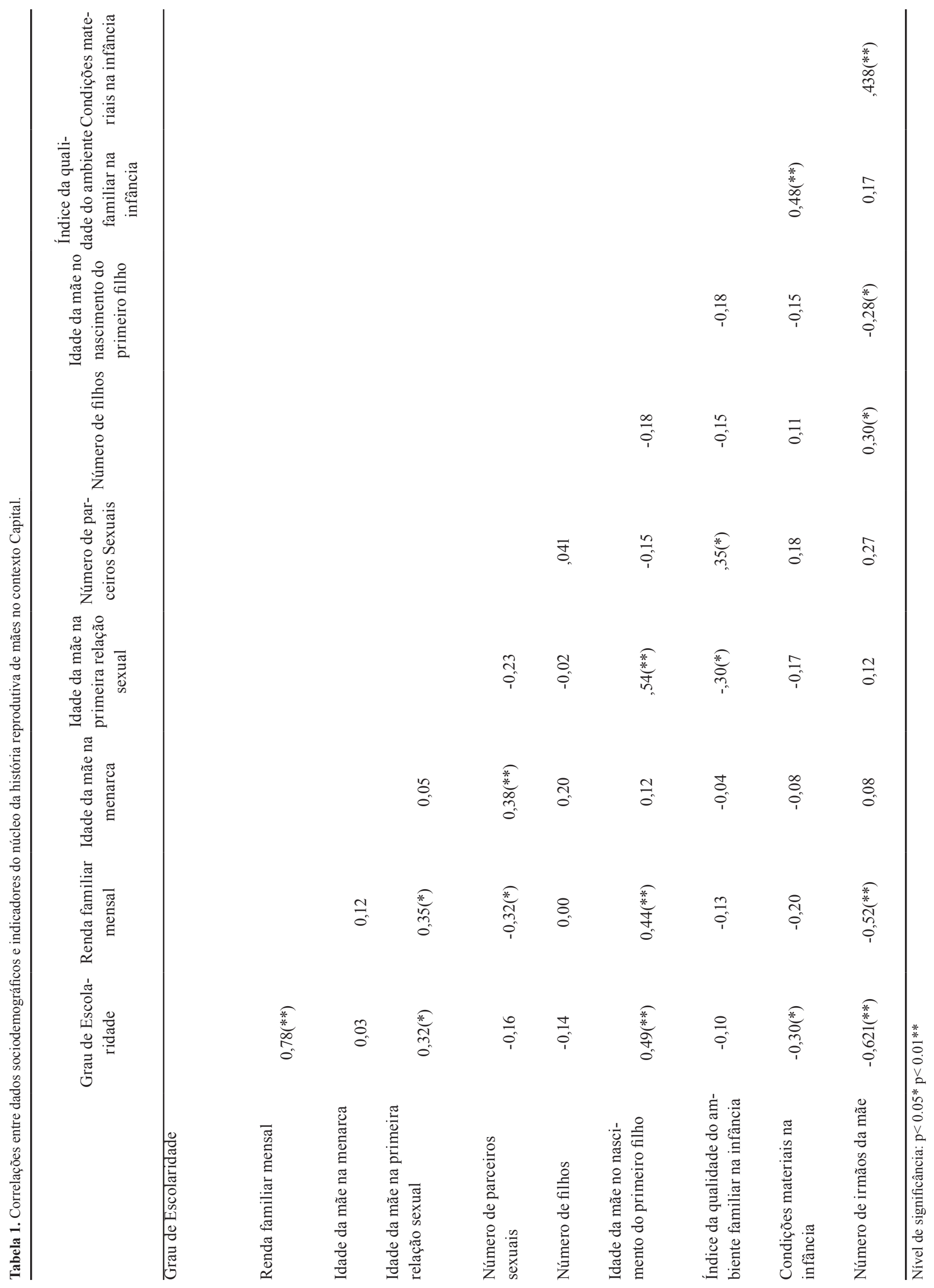




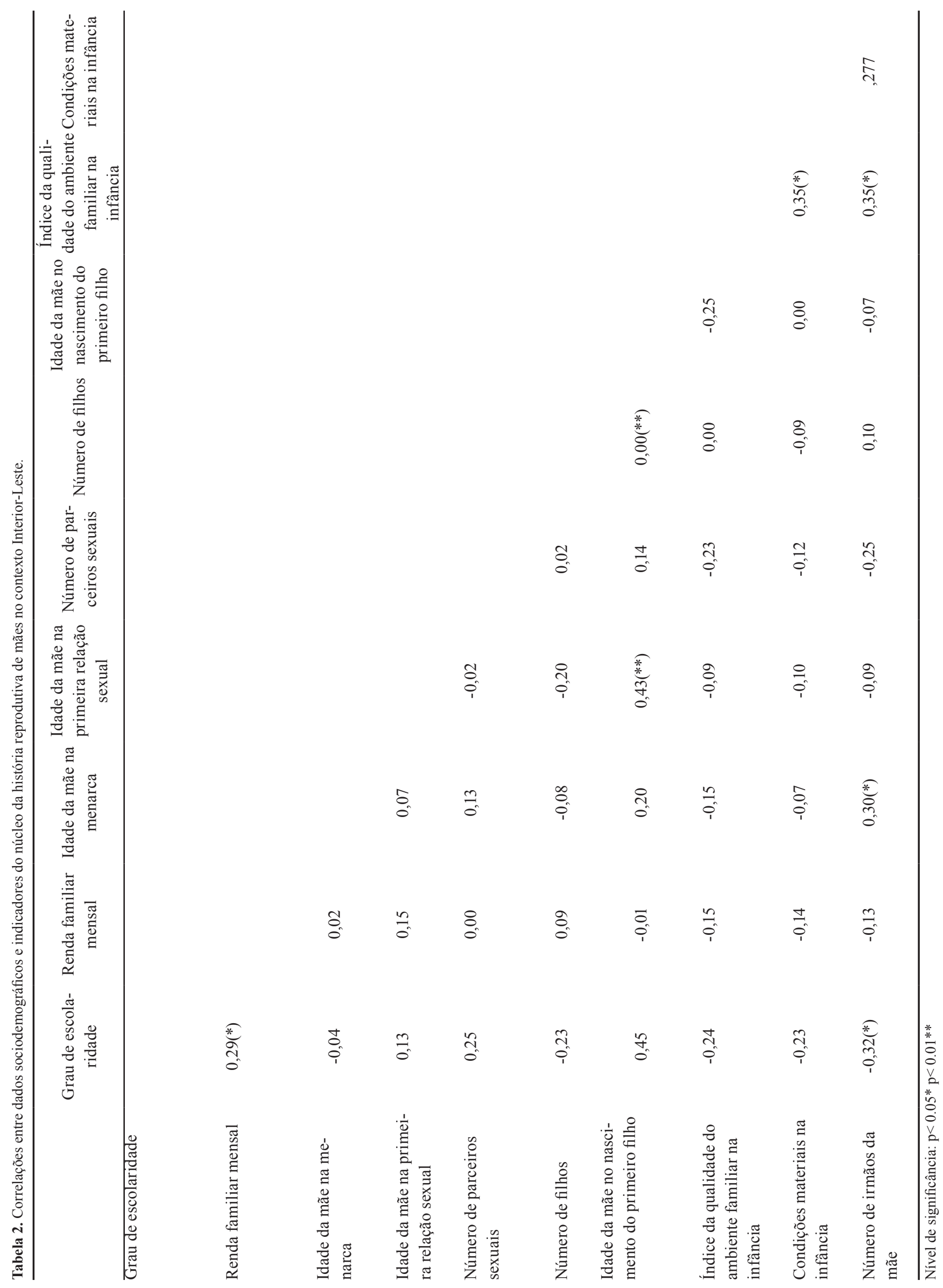




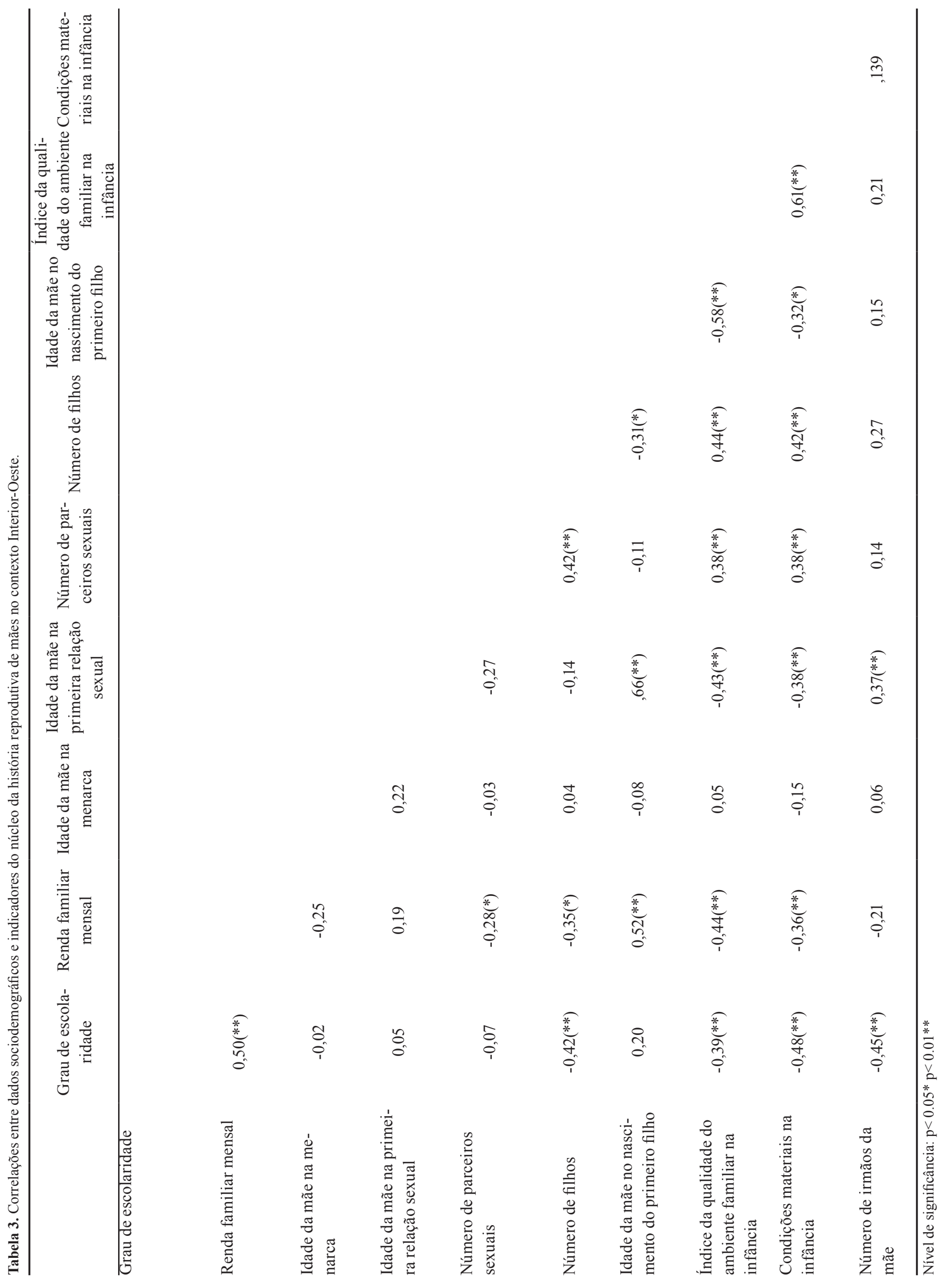


diretas entre a menarca e os demais núcleos, além de outros fatores (Kim \& Smith, 1998; Kim, Smith \& Palermiti, 1997; Maestripieri \& cols., 2004), outras pesquisas não encontraram associações significativas que envolvam a menarca e núcleos reprodutivos (Mace, 2000; Quinlan, 2003).

No presente estudo, a menarca também apareceu praticamente isolada dos demais núcleos, com exceção do contexto Capital, como pode ser visto na Tabela 1. Uma hipótese acerca da questão da menarca não ser um ponto pacífico entre as pesquisas coloca que esta é influenciada por hormônios ingeridos, estimulação audiovisual, genética e até mesmo fatores psicossociais, como divórcio, experiências de abuso, além de outros fatores ambientais (Graber, Brooks-Gunn \& Warren, 1995; Grivas, Vasiliadis, Mouzakis, Milvas \& Koufopoulos, 2006; Pasquet, 1999; Romans, Martin, Gendall \& Herbinson, 2003; Tavares \& cols., 2000).

Sobre a relação entre os núcleos reprodutivos com estresse e condições materiais, os contextos Capital e Interior-Leste apresentaram correlações. Porém, somente no Interior-Oeste as correlações ocorreram com mais de dois indicadores da história reprodutiva, indicando uma forte relação da estratégia sexual quantitativa com o ambiente estressante e com poucos recursos. Essa distinção do Interior-Oeste relaciona-se com um dos objetivos da pesquisa, em que se pretendia observar as diferenças das localidades estudadas e relacioná-las com as peculiaridades do contexto. Entretanto, as variáveis escolaridade e renda também apresentaram diferenças estatisticamente significativas entre os três contextos, principalmente no Interior-Oeste, além de forte correlação com os núcleos reprodutivos.

Como os resultados apontaram, constatou-se que nos três contextos foram encontradas relações entre o nível de escolaridade e renda familiar mensal com dados da trajetória reprodutiva, em maior ou menor grau. Em todas as correlações, verificou-se, também, que uma pior qualidade do ambiente familiar na infância estava diretamente associada com a estratégia de reprodução adotada pela mãe, seja essa estratégia qualitativa (mais relacionada com maiores níveis de escolaridade e renda, e baixo nível de estresse familiar na infância) ou quantitativa (mais relacionadas com baixa escolaridade e renda, e alto nível de estresse na vida familiar infantil). Esses dados parecem indicar que as estratégias adotadas não ocorrem ao acaso, mas que fatores da história de vida do indivíduo são elementos que exercem influência direta sobre essas escolhas, o que confirma a hipótese inicial do estudo de que o ambiente estaria relacionado com a estratégia reprodutiva adotada. A partir desses resultados fica evidente a importância do cuidado parental na infância, pois é nesse período que o indivíduo tem a oportunidade de acurar o potencial reprodutivo, além de perceber e interpretar corretamente os sinais do ambiente (Hill \& Kaplan, 1999; MacDonald, 1997; Mace, 2000; Maestripieri \& cols., 2004; Quinlan, 2003). Como já apontado em outras pesquisas, o cuidado parental reflete-se na vida adulta do indivíduo, inclusive no modo como este exercerá sua parentalidade (Cecconello, Antoni \& Koller, 2003).

Dentre os contextos, evidencia-se o Interior-Oeste, onde as correlações ocorreram mais entre os núcleos reprodutivos, além de resultarem em valores maiores, indicando correlações mais fortes entre as variáveis. Esse local de pesquisa destaca-se dos demais também em relação aos dados sociodemográficos e às características culturais e ecológicas. Possivelmente, a interação entre essas variáveis é responsável pela diferenciação do contexto em questão.

O destaque do Interior-Oeste pode ser explicado por meio da Teoria da História de Vida, em que os recursos e sinais do ambiente são interpretados pelo indivíduo e orientam suas estratégias de ação (Hill \& Kaplan, 1999; MacDonald, 1997; Mace, 2000; Maestripieri \& cols, 2004; Quinlan, 2003; Vigil, Geary \& Byrd-Craven, 2005). Como nesse contexto os níveis de urbanização, renda e escolaridade têm médias significativamente mais baixas, os recursos ambientais também são mais escassos e, por conseguinte, mais suscetíveis ao estresse. Como hipotetizado neste estudo, foi encontrado um pior índice de qualidade familiar passado e uma abordagem reprodutiva mais quantitativa nas mulheres do Interior-Oeste, em comparação com os outros contextos.

Ainda em relação aos contextos, verifica-se que os dados sociodemográficos parecem acompanhar o nível de urbanização: índices mais altos de escolaridade e renda apareceram no contexto Capital, que possui também o maior nível de urbanização, seguido pelo Interior-Leste e pelo Interior-Oeste, como descrito na seção do Método. Além de características biológicas e ecológicas, as diferenças culturais entre os contextos são fatores que também afetam aspectos do cuidado parental, da escolha de parceiros e demais estratégias de reprodução. Nesse sentido, a interação entre várias perspectivas, como sugere a Teoria da História de Vida, mostra-se importante e necessária para melhor compreensão do ser humano nos seus mais diversos ambientes.

A partir dos resultados deste estudo, surge outra questão: as variáveis escolaridade e renda estariam mais fortemente relacionadas com a estratégia reprodutiva ou sinais apresentados pelo ambiente cultural por si só responderiam sobre as diferenças entre os três contextos? Alguns estudos já apontam a importância das variáveis escolaridade e renda (Bem \& Wagner, 2006; Seidl-de-Moura \& cols., 2004; Silva, Vieira, Seidl-de-Moura \& Ribas Jr., 2005; Ribas Jr., Seidl-de-Moura \& Bornstein, 2007). Contudo, novos estudos investigando essas relações ainda necessitam ser realizados. Uma possibilidade seria realizar uma pesquisa no contexto Capital, porém selecionando uma amostra com escolaridade e renda aproximadas ao contexto InteriorOeste, ou seja, com níveis mais baixos. Isso contribuiria também para um aumento dos estudos no âmbito nacional que utilizem a Teoria da História de Vida, pois tais estudos ainda se encontram escassos.

\section{Referências}

Bem, L. A., \& Wagner, A. (2006). Reflexões sobre a construção da parentalidade e o uso de estratégias educativas em famílias de baixo nível socioeconômico. Psicologia em Estudo, 11, 63-71.

Bjorklund, D. F., \& Pellegrini, A. D. (2000). Child development and evolutionary psychology. Child Development, 71, 1687-1708.

Cecconello, A. M., Antoni, C., \& Koller, S. H. (2003). Práticas educativas, estilos parentais e abuso físico no contexto familiar. Psicologia em Estudo, 8, 45-54. 
Dancey, C. P., \& Reidy, J. (2006). Estatística sem matemática para a psicologia. Porto Alegre: Artmed.

Davis, J. N., \& Daly, M. (1997). Evolutionary theory and the human family. The Quarterly Review of Biology, 72, 407-435.

Ellis, B., \& Garber, J. (2000). Psychosocial antecedents of variation in girls' pubertal timing: Maternal depression, stepfather presence, and marital and familiar stress. Child Development, 71, 485-501.

Geary, D. C. (2000). Evolution and proximate expression of human paternal investment. Psychological Bulletin, 126, 55-77.

Geary, D. C., \& Flynn, M. V. (2001). Evolution of human parental behavior and human family, Parenting: Science and Practice, 1, 55-61.

Governo do Estado de Santa Catarina (2007). Municipios. Retirado em 13/08/07, de http://www.sc.gov.br/conteudo/ municipios/frametsetmunicipios.htm.

Graber, J. A., Brooks-Gunn, J., \& Warren, M. P. (1995). The antecedents of menarcheal age: Heredity, family environment, and stressful life events. Child Development, 66, 346-359.

Grivas, T. B., Vasiliadis, E., Mouzakis, V., Milvas, C., \& Koufopoulos, G. (2006). Association between adolescent idiopathic scoliosis prevalence and age at menarche in different geographic latitudes Scoliosis, 1, 1-12.

Hill, K., \& Kaplan, H. (1999). Life history traits in humans: Theory and empirical studies. Annual Review of Anthropology, 28, 397-430.

Hoier, S. (2002). Father absence and age at menarche: A test of four evolutionary models. Human Nature, 14, 209-233.

Hrdy, S. B. (2001). Mãe Natureza: uma visão feminina da evolução - maternidade, filhos e seleção natural (A. Cabral, Trad.). Rio de Janeiro: Campus (Trabalho original publicado em 1999).

Instituto Brasileiro de Geografia e Estatística - IBGE (2006). Cidades. Retirado em 13/08/07, de http://www.ibge.gov.br.

Keller, H. (1996). Evolutionary perspectives. Em J. W. Berry, Y. H. Poortinga \& J. Pandey (Orgs.), Handbook of cross-cultural psychology, Vol 1, Theory and method (pp. 215-256). Boston: Allyn and Bacon.

Keller, H. (2002). Development as the interface between biology and culture: A conceptualization of early ontogenetic experiences. Em H. Keller, Y. H. Poortinga \& A. Schölmerich (Orgs.), Between culture and biology: Perspectives on ontogenetic development ( $\mathrm{pp}$. 215-240). Cambridge: Cambridge University Press.

Kim, K., \& Smith, P. K. (1998). Childhood stress, behavioural symptoms and mother-daughter pubertal development. Journal of Adolescence, 21, 231-240.

Kim, K., Smith, P. K., \& Palermiti, A. L. (1997). Conflict in the childhood and reproductive development. Evolution and Human Behavior, 18, 109-142.

MacDonald, K. (1997). Life history and human reproductive behavior: Enviromental/contextual influences and heritable variation. Human Nature, 8, 327-359.
Mace, R. (2000). Evolutionary ecology of human life history. Animal Behavior, 59, 1-10.

Mace, R., \& Sear, R. (1997). Birth interval and the sex of children in a traditional African population: An evolutionary analysis. Journal of Biosocial Science, 29, 499-507.

Maestripieri, D., Roney, J. R., DeBias, N., Durante, K. M., \& Spaepen, G. M. (2004). Father absence, menarche and interest in infants among adolescent girls. Developmental Science, 7, 560-566.

Pasquet, P. (1999). Age at menarche and urbanization in Cameroon: Current status and secular trends. Annals of Human Biology, 26, 89-97.

Quinlan, R. J. (2003). Father absence, parental care, and female reproductive development. Evolution and Human Behavior, 24, 376-390.

Ribas Jr., R. C., Seid-de-Moura, M. L., \& Bornstein, M. H. (2007). Cognições maternas acerca da maternidade e do desenvolvimento humano: uma contribuição ao estudo da psicologia parental. Revista Brasileira de Crescimento e Desenvolvimento Humano, 17, 104-113.

Romans, S. E., Martin, J. M., Gendall, K., \& Herbinson, G. P. (2003). Age of menarche: The role of some psychosocial factors. Psychological Medicine, 33, 933-939.

Seidl-de-Moura, M. L., Ribas Jr., R. C., Piccinini, C. A., Bastos, A. C. S., Magalhães, C. M. C., Vieira, M. L., Salomão, N. M. R., Silva, A. M. P. M., \& Silva, A. K. (2004). Conhecimento sobre desenvolvimento infantil em mães primíparas de diferentes centros urbanos do Brasil. Estudos de Psicologia (Natal), 9, 421-429.

Silva, A. K., Vieira, M. L., Seidl-de-Moura, M. L., \& Ribas Jr., R. C. (2005). Conhecimento de mães primíparas sobre desenvolvimento infantil. Um estudo em Itajaí, SC. Revista Brasileira de Crescimento e Desenvolvimento Humano, 15, 01-10.

Simpson J. A., \& Gangestad, W. S. (2001). The evolution of human mating: Trade-offs and strategic pluralism. Behavioral and Brain Sciences, 23, 573-587.

Tavares, C. H. F., Haeffner, L. S. B., Barbieiri, M. A., Bettiol, H., Barbieri, M. R., \& Souza, L. (2000). Idade da menarca em escolares de uma comunidade rural do sudeste do Brasil. Caderno de Saúde Pública, 16, 709-715.

Vigil, J. M., Geary, D. C., \& Byrd-Craven, J. (2005). A life history assessment of early childhood sexual abuse in women. Developmental Psychology, 41, 553-561.

Voland, E. (1998). Evolutionary ecology of human reproduction. Annual Review of Anthropology, 27, 347-374.

Recebido em 19.05.2008 Primeira decisão editorial em 14.02.2009 Versão final em 10.05.2010 Aceito em 10.05.2010 